\title{
Atomic Structure of the Stoichiometric GaAs(114) Surface
}

\author{
J. Márquez, P. Kratzer, L. Geelhaar, K. Jacobi,* and M. Scheffler \\ Fritz-Haber-Institut der Max-Planck-Gesellschaft, Faradayweg 4-6, D-14195 Berlin, Germany
}

(Received 6 June 2000)

\begin{abstract}
The stoichiometric GaAs(114) surface has been prepared using molecular beam epitaxy followed by annealing in ultrahigh vacuum. Based on in situ scanning tunneling microscopy measurements and first-principles electronic-structure calculations, we determine the surface reconstruction which we call $\alpha 2(2 \times 1)$. Contrary to what is expected for a high-index surface, it is surprisingly elementary. The $(2 \times 1)$ unit cell contains two As dimers and two rebonded Ga atoms. The surface energy is calculated as $53 \mathrm{meV} / \AA^{2}$, which falls well within the range of low-index GaAs surface energies.
\end{abstract}

DOI: $10.1103 /$ PhysRevLett.86.115

The self-organized formation of low-dimensional quantum structures, by depositing a few monolayers of one semiconductor material onto another one with a different lattice constant, has attracted great interest due to the enormous potential of these nanostructures for technological applications [1]. Particularly, lasing devices operating with InAs quantum dots (QDs) that form on a GaAs(001) substrate have already been demonstrated [2]. The ongoing improvement of these devices is limited by the lack of a detailed knowledge of the underlying mechanisms yielding the formation of the nanostructures [3]. One of the difficulties that have to be overcome is related to the exact shape of the QDs, which is an important input factor for theoretical calculations [4-10]. So far, most theoretical calculations have assumed that only surfaces with low Miller indices occur as bounding facets of the QDs [8-10]. However, experiments have revealed that also facets having high Miller indices $(\{113\},\{114\}[11-13],\{102\}[14]$, $\{104\}[15],\{125\}$ [13], and $\{136\}[16])$ might occur. These observations led to the question of whether the respective surfaces are stable and thereby contribute to an equilibrium structure [9], or whether the facets occur only because of growth kinetics [17]. In order to elucidate this problem it is necessary to study directly these surfaces. So far, among these QD-related surfaces the only one whose structure has been determined is $\operatorname{GaAs}(113) A(8 \times 1)[3,18]$.

Of particular interest is the (114) orientation. Because of its similarity to (001), the GaAs(114) surface has been considered in early studies of heterostructures as a highly vicinal $\mathrm{GaAs}(001)$ surface, whose terraces extend only over a single $\beta(2 \times 4)$-unit cell $[19,20]$. Moreover, this stepped morphology led to the assumption that this surface should be predestinated to accommodate strain in heterostructures [21].

Besides these studies on heterostructures grown on GaAs(114) surfaces, only few studies have been performed on the bare GaAs(114) surface so far. Under As-rich preparation conditions the surface revealed an atomically rough structure, whose reconstruction model was derived from reconstructions known for the GaAs(001) surface [19,22-24]. However, under Ga-rich conditions a transition to an extremely flat surface has been reported
PACS numbers: 68.35.Bs, 61.16.Ch, 61.50.Ah, 81.05.Ea

$[24,25]$. Based on scanning tunneling microscopy (STM) the respective authors proposed structural models which are highly questionable, since they contain surface atoms having two dangling bonds each. In contrast, for most reconstructed surfaces the average number of dangling bonds per surface atom is strongly (sometimes below one) reduced [26].

In this Letter we report on the GaAs(114) surface, prepared under Ga-rich conditions by molecular beam epitaxy (MBE). We have performed in situ STM measurements combined with first-principle electronic structure calculations, in order to determine the atomic structure of the surface. We show that GaAs(114) possesses a well-ordered reconstruction, which we call $\alpha 2(2 \times 1)$. Contrary to the large and complicated $\mathrm{GaAs}(113) \mathrm{A}(8 \times 1)$ reconstruction unit cell, which extends laterally over eight lattice spacings and vertically over six atomic layers, the unit cell of the $\operatorname{GaAs}(114) \alpha 2(2 \times 1)$ surface is surprisingly simple.

Experiments were carried out in a multichamber UHV system, which is described in detail elsewhere [27]. Samples with a typical size of $10 \times 10 \mathrm{~mm}^{2}$ were cut from GaAs(114) wafers ( $n$-type, Si-doped, carrier concentration $2.5 \times 10^{18} \mathrm{~cm}^{-3}$, Wafer Technology), cleaned with propanole, and introduced into the UHV system via a loading chamber. After oxide desorption at $580^{\circ} \mathrm{C}$, samples were treated with several ion-bombardment-andannealing cycles. Undoped layers $50-100 \mathrm{~nm}$ thick were grown by MBE at a temperature of $560{ }^{\circ} \mathrm{C}$. The $\mathrm{As}_{2}: \mathrm{Ga}$ beam equivalent pressure ratio was 20 . During growth the surface quality was reported by reflection high-energy electron diffraction (RHEED). In order to prepare the surface under Ga-rich conditions, the samples were annealed at $580{ }^{\circ} \mathrm{C}$ for $5 \mathrm{~min}$ without supplying any $\mathrm{As}_{2}$ flux and allowed then to cool for more than $1 \mathrm{~h}$. Surfaces were in situ studied by STM (Park Scientific Instruments, VP2) and low-energy electron diffraction (LEED). STM images were acquired in constant current mode.

Calculations of the geometric and electronic structure of the GaAs(114) surface were performed within the localdensity approximation by employing a plane-wave pseudopotential approach [28] with a cutoff energy of 10 Ryd. The GaAs(114) surface was modeled using periodic slabs 
of $\sim 12 \AA$ GaAs, separated by a vacuum region of $10 \AA$. The lowest atoms in the slab had their dangling bonds saturated with pseudohydrogen atoms and were held fixed, while the surface and subsurface atoms were relaxed until all the forces were less than $0.1 \mathrm{eV} / \AA$. After structural relaxation, isocontour surfaces of a suitably defined local density of states were extracted. To simulate filled state (negative sample bias) STM images, the local density of valence states was integrated from the valence band maximum to $2.5 \mathrm{eV}$ below. The isocontour surfaces generated in this way correspond to STM images taken in constant current mode [29].

An experimental filled-state STM image is shown in Fig. 1. The most prominent features are the rows of bright double spots along [110]. The separation between the double spots in direction [1110] is $8.3 \pm 0.9 \AA$ corresponding to twice the length of the bulktruncated unit cell of $4.0 \AA$. In the [221] direction the rows are separated by $17.2 \pm 0.9 \AA$ which is equal to the length of the bulk-truncated unit cell of $17.0 \AA$. A $(2 \times 1)$ reconstruction unit cell is found as indicated Fig. 1. With respect to size and orientation, The unit cell is in excellent agreement with LEED images of the surface (not shown here). It is well known that the electronic states lying $0-3 \mathrm{eV}$ below the valence band maximum are mainly derived from As, in the bulk as well as at the surface of GaAs. Since the sample is biased negatively with respect to the tip, we know that we image these As-derived states. In our model discussed below the double spots are identified as As dimers. Most remarkably the two As atoms forming the dimer are well resolved. To our knowledge this has not been achieved for any other GaAs surface, not even for the extensively studied $\operatorname{GaAs}(001) \beta 2(2 \times 4)$ surface. The area between

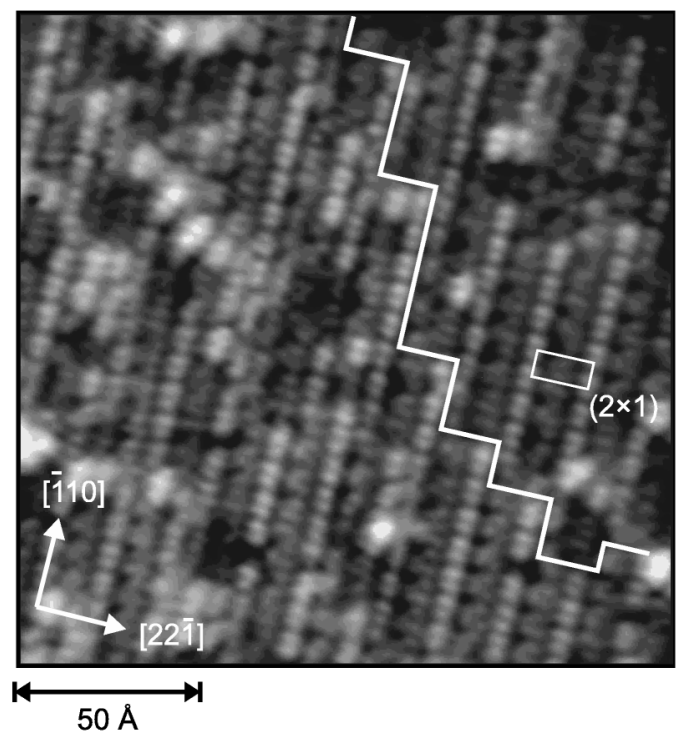

FIG. 1. STM image of the stoichiometric GaAs(114) $\alpha 2(2 \times$ 1) surface. $U_{\text {sample }}=-2.5 \mathrm{~V}, I=0.2 \mathrm{nA}$. A step edge and a $(2 \times 1)$ unit cell are marked by white lines. the dimer rows exhibits only weak intensity which is inhomogeneously distributed. At the right-hand side of the dimer rows "black holes" are observed showing often also a $2 \times$ periodicity. At the left-hand side of the dimer rows the intensity is much more uniform.

Looking in detail at Fig. 1, five terraces can be recognized due to some unintended misorientation of the sample. The step height amounts to about $1.3 \AA$ which is much less than its value on $\operatorname{GaAs}(001)$ (2.8 $\AA$ ). Also, we have subtracted a slope in order to achieve constant contrast throughout the figure. For these reasons the steps are difficult to be recognized in Fig. 1. Therefore, we have marked one step in the middle to better guide the eye.

In order to set up a model we consider the bulk-truncated surface as depicted in Fig. 2. The uppermost atomic plane contains atoms $\left(1,1^{\prime}, 5,5^{\prime}, 6,6^{\prime}\right)$, the second plane $\left(2,2^{\prime}\right.$, $\left.3,3^{\prime}\right)$, etc. Atom (4) lies in the third plane. First, one recognizes that each plane contains the same number of $\mathrm{Ga}$ and As atoms so that the surface is stoichiometric. The biggest step in reducing the surface energy is the formation of dimers, especially of As dimers. So, the As atoms $\left(1,1^{\prime}\right),\left(3,3^{\prime}\right)$, and $\left(6,6^{\prime}\right)$ may form dimers. This already fulfills two major requirements from the experimental results: a $2 \times$ period is created along [ $[110]$ as well as a row of high lying As dimers. From Fig. 2(b) it is also obvious how one may further reduce the surface energy: by forming a Ga-Ga bond between atoms (4) and (5), atom (5) gets
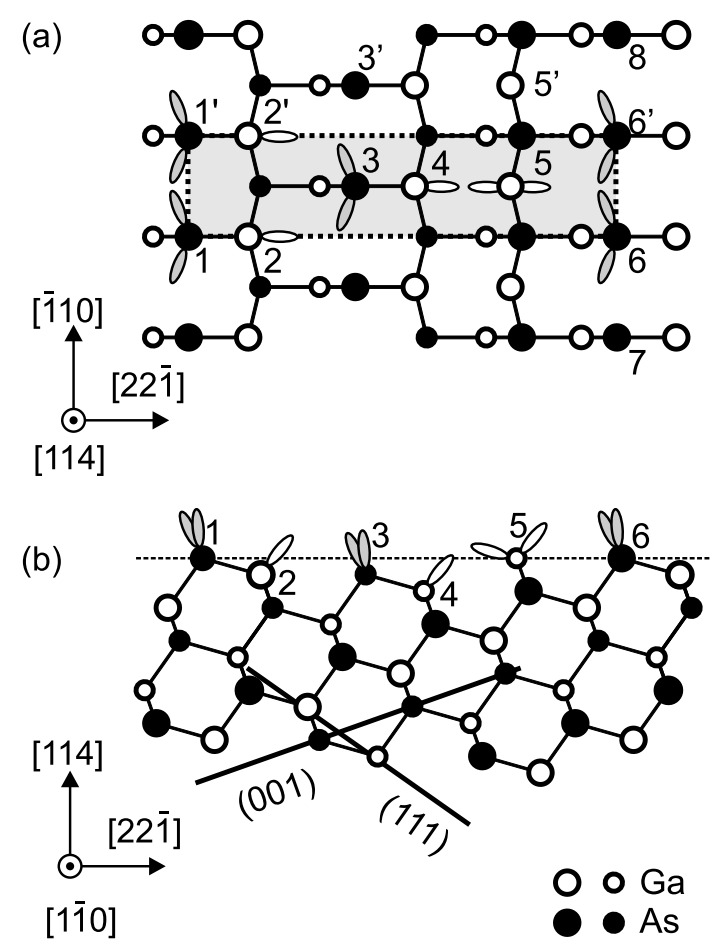

FIG. 2. Schematic sketch of the bulk truncated GaAs(114) surface. (a) Top view. The $(1 \times 1)$ unit cell is marked by the gray rectangle. For the surface atoms in the unit cell the dangling bonds are indicated. The topmost atoms (surface atoms) are drawn in larger size. (b) Side view. The size of the atoms varies with the height perpendicular to the figure plane. 
rebonded and one reduces the number of DBs from 3 to 1 at this group. For reasons given below, we call this model $\alpha 2(2 \times 1)$. Per $(2 \times 1)$ unit cell there are four As dangling bonds from the dimers and four empty Ga dangling bonds from atoms 2 and 5 and their analogs. On account of this, the $\alpha 2(2 \times 1)$ reconstruction fulfills the electron counting rule [30] which simply states that a semiconductor surface is stabilized if it is free of electronic states in the fundamental band gap. As a necessary condition, the electrons in the partially filled broken bonds at the surface must be redistributed so that anion dangling bonds get filled and the cation bonds emptied. Figure 2 also shows that the Ga dangling bonds are oriented perpendicular to [110] and thus any periodicity doubling along [1110] could not be caused by Ga dimers but only by As dimers.

The above arguments make the proposed $\alpha 2(2 \times 1)$ model a very likely candidate for the observed structure. To demonstrate the correctness of this model, we have performed total energy calculations for this and other reasonable models, among them the $\alpha(2 \times 1)$ model as proposed by Yamada et al. [24]. The main support to the $\alpha 2(2 \times 1)$ model derives from its thermodynamical stability, as inferred from calculated surface energies. For Ga-rich preparation conditions, the calculations showed that the $\alpha 2(2 \times 1)$ structure has the lowest surface energy of all models considered, $53 \AA^{2}$, independent of the As chemical potential. Although the $\alpha 2(2 \times 1)$ reconstruction appears under Ga-rich preparation conditions, it contains enough As atoms at the surface to be stoichiometric. The surface energy for stoichiometric surfaces is generally independent of the chemical environment (see, e.g., Ref. [31]). The calculated value for the surface energy is well within the range of those found for low-index GaAs surfaces [31]. Moreover, the GaAs(114) $\alpha 2(2 \times 1)$ reconstruction is of lower surface energy than the well-known $\mathrm{GaAs}(001) \beta 2(4 \times 2)$ reconstruction $(64 \mathrm{meV} / \AA 2)$ [31] under Ga-rich conditions.

The relaxed atomic geometry for the $\alpha 2(2 \times 1)$ model as obtained from the calculations is displayed in Figs. 3(a) and 3(b). Furthermore, we have calculated a constantcurrent STM image as shown in Fig. 3(c). We learn from the simulation that the STM image quite closely reflects the topography of the surface. The As dimers give rise to the highest occupied surface state. In addition, the higher dimer row is the most prominent feature since it is the highest topographic feature. The low intensity in between strongly favors our model in which this region is dominated by $\mathrm{Ga}$ atoms. The $\mathrm{Ga}$ atoms are hardly visible in the range of applied tip voltages, since their dangling bonds give rise only to empty surface states. The lower As dimers do not contribute very much either as they lie $0.7 \AA$ lower. For comparison, we present an enlarged STM picture in Fig. 3(d). The main topographic features, i.e., the higher As dimer rows, are clearly visible and, therefore, easy to recognize in both simulated and measured STM images, but the comparison of the features within the trench is more complicated. For this comparison one has to consider (a)

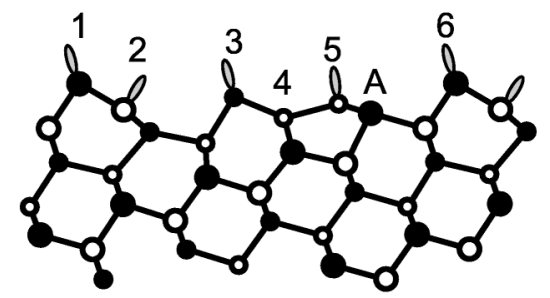

(b)
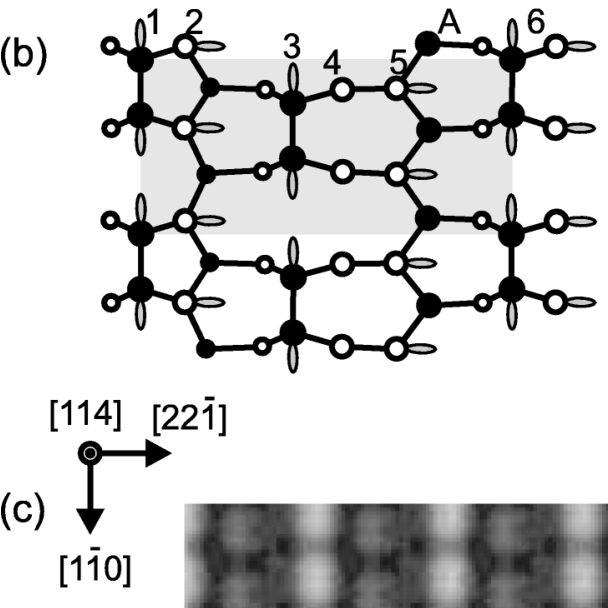

(d)
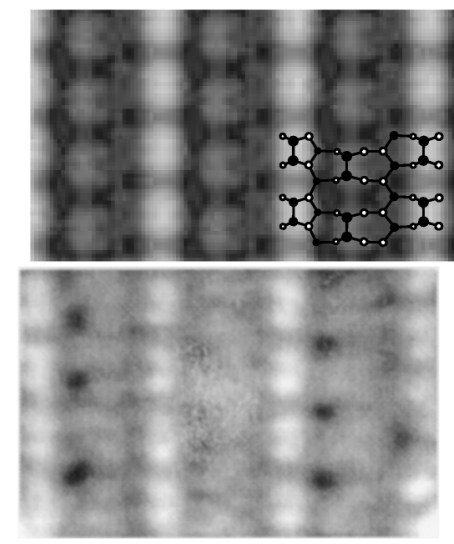

FIG. 3. Structure of the $\operatorname{GaAs}(114) \alpha 2(2 \times 1)$ surface as derived from $a b$ initio total energy calculations: (a) side view; (b) top view; (c) simulated STM image with an overlay of two $(2 \times$ 1) surface unit cells; (d) experimental STM image (parameters as for Fig. 1).

that there is some degree of disorder along [1110] within the trench, due to the fact that the As dimers may form shifted up- or downwards with respect to the topmost As dimers. A similar disorder in the arrangement of As dimers is observed in the trenches on $\operatorname{GaAs}(001) \beta 2(2 \times 4)$ [32]. In addition, even As dimer vacancies and single As atoms seem to occur.

With these considerations in mind, the intensity variations in the trench between the dimer rows can be understood. First, one notices that there is a shallow trench at the right-hand side of the topmost As dimer rows. This is well described in the model [Fig. 3(a)]: At the right-hand side, atom (2) is lowered due to its $s p^{2}$ rehybridization forming a trench between atoms (1) and (3), whereas at the left-hand side of the topmost As dimer row, the As atom (A) is moved upwards by the rebonded atom (5) filling up this side. Second, one recognizes features like the abovementioned "black holes" at the right-hand side of the upper 
dimers, which give rise to a $2 \times$ periodicity. This periodicity is caused by the As dimers (bond length $2.49 \AA$ ), which divide the row into filled and open sections and thereby reveal the black holes. In the rows of the black holes, there is sometimes a phase shift or another distortion. This can be understood assuming that some As atoms (3) are missing and/or that the lower dimers may not always be in the same phase with respect to the upper dimers.

The reconstruction yields a structure with a remarkably small corrugation in the unit cell, as can be seen in Fig. 3(a). The height difference between the highest surface atom (1) and the lowest (4) amounts to only $1.5 \AA$. For comparison, we note that the $\operatorname{GaAs}(001) \beta 2(2 \times 4)$ unit cell is much more corrugated, with a height difference between As dimers of $2.72 \AA$ [32]. The flatness of the (114) unit cell is mainly an intrinsic property of this crystallographic plane, but is augmented by the $\alpha 2(2 \times 1)$ reconstruction at the price of a significant elongation of the bonds.

Furthermore, we would like to comment on our nomenclature. From Fig. 2 one recognizes that there is still another positioning of the surface atoms possible which does not change the number and kind of surface atoms. One may lift the dimerization of atoms $\left(3,3^{\prime}\right)$, move atom (5) on top of atoms $\left(2^{\prime}, 3,3^{\prime}\right)$, and dimerize the As atoms underneath atom (5). This procedure would shift the rebonded $\mathrm{Ga}$ atom to the left and one As dimer to the right. A close inspection shows that this reconstruction is analogous to $\operatorname{GaAs}(001) \alpha(2 \times 4)$ [33] and has been denominated $(114) \alpha(2 \times 1)$ [24]. Therefore we call our structure $(114) \alpha 2(2 \times 1)$ and mention that it is the analog to $\operatorname{InAs}(001) \alpha 2(2 \times 4)$ [34]. On GaAs(001) the equivalent structure has not been observed.

Although the $\operatorname{GaAs}(114) \alpha(2 \times 1)$ model was supported by STM [24] and surface core level shifts in photoemission [23], we have demonstrated that actually the stoichiometric $\operatorname{GaAs}(114)$ surface is reconstructed according to the $\alpha 2(2 \times 1)$ model. The sharp rows of single As dimers clearly discriminate the $\alpha 2$ from the $\alpha$ structure, which has a double row of As dimers. The surface core-level shifts in photoemission indicating a Ga-Ga bond favored the $\alpha(2 \times 1)$ model against the As-rich model at that time [23]. Since the $\alpha 2(2 \times 1)$ contains the same kind of $\mathrm{Ga}-\mathrm{Ga}$ bonds it is not in conflict with the core-level-shift data.

In conclusion, this Letter presents the structure determination of the reconstructed, stoichiometric $\operatorname{GaAs}(114) \alpha 2(2 \times 1)$ surface. After GaAs$(113) \mathrm{A}(8 \times 1)$ $[3,18]$ this is only the second example of a stable, high-index GaAs surface for which the structure could be determined. The found reconstruction is surprisingly simple as only a change in rehybridization and a bond formation between neighboring atoms of the bulktruncated surface takes place. The calculated surface energy is $53 \mathrm{meV} / \AA 2$ independent of the As chemical potential. We call this model $\alpha 2(2 \times 1)$ in analogy to the notation for the (001) surfaces. We observed an atomically resolved STM picture in which the individual atoms of the As dimer are better resolved than for any other GaAs surface. The observed $\operatorname{GaAs}(114) \alpha 2(2 \times 1)$ surface is very smooth on an atomic scale which may explain to some extent the existence of very flat interfaces observed for (114)-based heterostructures.

We acknowledge the support by G. Ertl, DFG SFB 296, and BMBF 05622EBA4.

*Corresponding author.

Electronic address jacobi@fhi-berlin.mpg.de

[1] D. Bimberg, M. Grundmann, and N. N. Ledentsov, Quantum Dot Heterostructures (Wiley, Chichester, 1999).

[2] N. Kirstaedter et al., Electron. Lett. 30, 1416 (1994).

[3] J. Platen et al., J. Appl. Phys. 85, 3597 (1999).

[4] B. G. Orr et al., Europhys. Lett. 19, 33 (1992).

[5] C. Ratsch and A. Zangwill, Surf. Sci. 293, 123 (1993).

[6] C. Priester and M. Lannoo, Phys. Rev. Lett. 75, 93 (1995).

[7] Y. Chen and J. Washburn, Phys. Rev. Lett. 77, 4046 (1996).

[8] V. A. Shchukin et al., Phys. Rev. Lett. 75, 2968 (1995).

[9] N. Moll et al., Phys. Rev. B 58, 4566 (1998).

[10] L. G. Wang et al., Phys. Rev. Lett. 82, 4042 (1999).

[11] Y. Nabetani et al., J. Appl. Phys. 76, 347 (1994).

[12] B. A. Joyce et al., J. Vac. Sci. Technol. B 16, 2373 (1998).

[13] Y. Hasegawa et al., Appl. Phys. Lett. 72, 2265 (1998).

[14] H. Eisele et al., Surf. Interface Anal. 27, 537 (1999).

[15] J. M. Moison et al., Appl. Phys. Lett. 64, 196 (1994).

[16] H. Lee et al., Appl. Phys. Lett. 72, 812 (1998).

[17] D. E. Jesson et al., Phys. Rev. Lett. 80, 5156 (1998).

[18] M. Wassermeier et al., Phys. Rev. B 51, 14721 (1995).

[19] S. Shimomura et al., Jpn. J. Appl. Phys. 32, L1728 (1993).

[20] Y. Tsuda et al., J. Cryst. Growth 150, 415 (1995).

[21] A. Ponchet et al., Microelectronics J. 26, 783 (1995).

[22] S. Shimomura et al., J. Vac. Sci. Technol. B 13, 696 (1995).

[23] J. Platen et al., Appl. Surf. Sci. 123/124, 43 (1998).

[24] T. Yamada et al., J. Cryst. Growth 150, 421 (1995).

[25] H. Yamaguchi et al., Jpn. J. Appl. Phys. 34, L1490 (1995).

[26] C. B. Duke, Chem. Rev. 96, 1237 (1996).

[27] P. Geng et al., Rev. Sci. Instrum. 71, 504 (2000).

[28] M. Bockstedte et al., Comput. Phys. Commun. 107, 187 (1997).

[29] J. Tersoff and D. R. Hamann, Phys. Rev. B 31, 805 (1985).

[30] D. J. Chadi, in The Structure of Surfaces III, Springer Series in Surface Sciences, edited by S. Y. Tong et al., (Springer-Verlag, Berlin, Heidelberg, 1991), Vol. 24, p. 532.

[31] N. Moll et al., Phys. Rev. B 54, 8844 (1996).

[32] V. P. LaBella et al., Phys. Rev. Lett. 83, 2989 (1999).

[33] H. H. Farrell and C. J. Palmstrøm, J. Vac. Sci. Technol. B 8, 903 (1990).

[34] H. Yamaguchi and Y. Horikoshi, Jpn. J. Appl. Phys. 33, L1423 (1994). 